\title{
Gravitational Waves from Collapsing Domain Walls
}

\author{
Takashi Hiramatsu, ${ }^{1}$ Masahiro Kawasaki, ${ }^{1,2}$ and Ken'ichi Saikawa ${ }^{1}$ \\ ${ }^{1}$ Institute for Cosmic Ray Research, The University of Tokyo, \\ 5-1-5 Kashiwa-no-ha, Kashiwa City, Chiba 277-8582, Japan \\ ${ }^{2}$ Institute for Physics and Mathematics of the Universe, The University of Tokyo, \\ 5-1-5 Kashiwa-no-ha, Kashiwa City, Chiba 277-8582, Japan
}

\begin{abstract}
We study the production of gravitational waves from cosmic domain walls created during phase transition in the early universe. We investigate the process of formation and evolution of domain walls by running three dimensional lattice simulations. If we introduce an approximate discrete symmetry, walls become metastable and finally disappear. This process might occur by a pressure difference between two vacua if a quantum tunneling is neglected. We calculate the spectrum of gravitational waves produced by collapsing metastable domain walls. Extrapolating the numerical results, we find that the signal of gravitational waves produced by domain walls whose energy scale is around $10^{10}-10^{12} \mathrm{GeV}$ will be observable in the next generation gravitational wave interferometers.

PACS numbers: 98.80.Cq, 04.30.Db
\end{abstract}

\section{INTRODUCTION}

The spontaneous symmetry breaking is one of the most important ingredients of the modern particle physics. Not only it describes the unification of forces acting on the elementary particles, but also it has rich consequences in the early universe. One is the occurrence of primordial phase transitions followed by the formation of topological defects [1. Especially, if there was the spontaneous breaking of a discrete symmetry, the two dimensional surface-like defects, called domain walls, would be formed in the early era of the universe (for a review of domain walls and other topological defects, see [2]). Although discrete symmetries naturally arise in many particle physics models, the existence of stable domain walls is disfavored by cosmological considerations. Indeed, if domain walls survived until the present time, the energy density of walls would eventually come to dominate the total energy density of the universe, causing the fast expansion of the universe to affect the galaxy formation or primordial nucleosynthesis 3 . Moreover, the presence of domain walls would cause the excessive anisotropy in the cosmic microwave background observed today, which rules out the existence of stable domain walls with the symmetry breaking scale $\eta \gtrsim 1 \mathrm{MeV}$ [4.

However, it is still possible to consider the existence of unstable domain walls which decay early enough not to lead cosmological disasters. One way to introduce the instability of walls is to make discrete symmetry only approximate and tilt the potential so as to lift the degeneracy of vacua. This might be raised by nonrenormalizable operators suppressed by the cutoff scale ( $\sim$ Planck scale) [5]. The idea of an approximate discrete symmetry as a mechanism to remove the domain wall problem has been studied by several authors $3,6,8$. Also, there is another possibility to create unstable domain walls due to a postinflationary nonthermal phase transition 9 , 10.

In this paper we investigate the production of gravitational waves from the annihilating process of unstable domain walls. There are early works studying the bubble collisions in the early universe [11] and the possibility of generating gravitational waves [12 16. In these works, gravitational waves are considered to be generated by the collision of spherically expanding bubbles, which are created during first order phase transitions (i.e. the quantum tunneling from the false vacuum to the true vacuum). Instead, in this paper we assume that the transition from one vacuum to another proceeds via a realignment of the classical field rather than a quantum tunneling. The production of gravitational waves from such a process was originally calculated in [17]. However, in [17] the radiated energy is calculated only in the simple configuration such as situations with axial symmetry, and only the total intensity of gravitational waves was estimated. Instead, our purpose is to study the production of gravitational waves in more realistic situations (i.e. to follow from phase transition and to consider generic configuration of the scalar field), and calculate the spectrum of gravitational waves.

The gravitational wave is the powerful tool for studying cosmology and high energy physics (for reviews, see [18 20]). There are various ongoing and planned experiments on the gravitational waves. As for the ground-based experiment, LIGO [21] is running, and LCGT [22] is planned. And space-borne interferometers such as LISA [23], BBO [24], and DECIGO [25] are planned to be launched in the future. Furthermore, it has been started to plan the ground-based interferometer with improved sensitivity, "Einstein Telescope(ET)" [26] in Europe recently. In this paper we discuss whether the stochastic gravitational wave backgrounds produced by domain walls are detectable in these experiments. If it is detectable and the predicted form of the spectrum is distinctive, we expect that the gravitational wave spectrum from domain walls can be another probe for high energy physics. One example is in the theory with supersymmetry. One may be able to measure the parameter of the theory such as the mass of the gravitino, fermionic partner of the 
graviton, by gravitational waves from annihilation of $Z_{2 N}$ domain walls 27 .

The dynamics of domain walls is not easy to investigate because of the nonlinear nature of the evolution equations. Thus it is necessary to use numerical calculations to follow the evolution of domain walls with arbitrary structures. The first complete numerical study was performed by Press, Ryden, and Spergel 28] by using the modified field equation for the scalar field (called the PRS algorithm). Also, Kawano 29] simulated the evolution of domain walls by using the thin wall effective equation of motion (similar to the Nambu-Goto equation [30, 31] for cosmic strings). Later, several authors investigated further by up-dated high resolution simulations 32 34 and the numerical simulations for unstable domain walls are performed in [10, 35. However, although these numerical simulations successfully demonstrate the macroscopic evolution of domain wall networks, they put an unphysical assumption about the width of a domain wall, and we cannot use their computational method since this method may invalidates the estimation of the energy of gravitational waves (see Section IV A). Therefore, we solve the field equations directly rather than rely on the previous methodologies. This gives us severe technical restrictions in choosing parameters of the simulations.

This paper is organized as follows. In Section [I] we shortly review the dynamics of domain wall networks. In Section [II] we describe the formalism to calculate classical gravitational wave spectra radiated by the scalar field in the systematic way. Then, after a short discussion about the difficulty in numerical simulations, we present the results of our numerical simulations in Section IV]. We combine the results of numerical calculations with analytic estimations, and discuss the observability of the spectrum by extrapolating them in Section $\mathrm{V}$. Finally we conclude in Section VI

\section{DOMAIN WALL DYNAMICS}

\section{A. The Model of Domain Walls}

We consider the model of the real scalar field with the potential

$$
V(\phi)=\frac{\lambda}{4}\left(\phi^{2}-\eta^{2}\right)^{2}+\epsilon \eta \phi\left(\frac{1}{3} \phi^{2}-\eta^{2}\right)+\frac{\lambda}{8} T^{2} \phi^{2} .
$$

The first term is the usual double well potential. In the absence of the second term, this model has a discrete $Z_{2}$ symmetry corresponding to the transformation $\phi \rightarrow-\phi$. The second term is introduced in order to lift the degeneracy of the two vacua and make the discrete symmetry approximate. In this model, the scalar field is assumed to be thermal equilibrium at the first stage of their evolution, and we introduce the finite temperature correction in the last term of the potential. When finite temperature effects become negligible, the potential has two minima $\phi= \pm \eta$ and the formation of domain walls occurs. The difference of the energy density between two minima is $\Lambda=4 \epsilon \eta^{4} / 3$ and the height of the potential barrier is $\Delta V \simeq \lambda \eta^{4} / 4$. The dimensionless constant parameter $\epsilon$ is called "bias", which makes domain walls unstable.

In the numerical simulations which we will describe later, we solve the classical filed equation with the potential given by Eq. (1). This corresponds to the fact that we consider only a quiescent second order transition. If there are more violent first order transitions via a quantum tunneling, the gravitational wave signature might be enhanced significantly. Such a quantum treatment is, however, beyond the scope of this paper.

In the following two subsections, we briefly review the property of the evolution of domain wall networks.

\section{B. Scaling Solutions}

The well known property of the evolution of domain wall networks is that there exists the phase of "scaling", in which the typical length scales such as the wall curvature radius $R$ and the distance of two neighboring walls $L$ are given by Hubble radius,

$$
R \sim L \sim H^{-1} \sim t
$$

This property is originally studied in [28 numerically, and by other groups in [10, 32 35]. However, the analytic modeling of the scaling solution is found in 36 38.

When domain wall networks are in scaling regime, the energy density of domain walls is given by

$$
\rho_{w} \sim \sigma R^{2} / R^{3} \sim \sigma / t
$$

where $\sigma=2 \sqrt{2 \lambda} \eta^{3} / 3$ is the tension of the wall. Eq. (3) is also expressed as

$$
A / V \propto \tau^{-1}
$$


where $A / V$ is the comoving area density of the wall, and $\tau$ is the conformal time: $d \tau=d t / a$. If we assume radiation dominated universe, $A / V$ is proportional to $t^{-1 / 2}$, which is a criterion to distinguish whether walls are in scaling regime.

\section{The Role of the Bias}

There are two effects caused by the bias in the potential. One is the bias of two vacua chosen during the phase transition, and another is the volume pressure which shrinks the false vacuum domains and eliminates domain walls. The cosmological scenario with biased discrete symmetry is investigated in [3] and here we follow the analysis of it.

Let us denote the probability of having a scalar field fluctuation during the phase transition end up in plus vacuum as $p_{+}$and in minus vacuum as $p_{-}$. If there exists the bias, the ratio of these two probabilities is given by

$$
\frac{p_{+}}{p_{-}}=\exp \left(-\frac{\Delta F}{T}\right) .
$$

Here, $\Delta F=\Lambda \times \xi_{\text {corr }}^{3}$ is the difference of the free energy between two vacua, and $\xi_{\text {corr }}$ is the correlation length of the scalar fields. If we estimate $T$ as Ginzburg temperature [1, 3], given by $T_{G} \simeq \Delta V \times \xi_{\text {corr }}^{3} \simeq \lambda \eta^{4} \xi_{\text {corr }}^{3} / 4$, the above equation can be written as

$$
\frac{p_{+}}{p_{-}}=\exp \left(-\frac{\Lambda}{\Delta V}\right) \simeq \exp \left(-\frac{16}{3} \frac{\epsilon}{\lambda}\right) .
$$

Therefore, there exists the bias between two vacua if $\epsilon \neq 0$. The spatial distribution of two vacua after phase transition has been studied by using percolation theory [39]. It has been shown that if the probability $p_{+}$(or $p_{-}$) is bigger than a critical value $p_{c}$, an infinite plus (minus) cluster appears in the space. If we approximate the space as a three dimensional cubic lattice, $p_{c}=0.311$ [39], and by requiring both $p_{+}$and $p_{-}$are bigger than $p_{c}$, we obtain

$$
\epsilon<0.15 \lambda \text {. }
$$

If the condition given by Eq. (7) is satisfied, the infinite size of domain walls would appear after phase transition.

After the formation of domain walls, there are two forces acting on the walls. The first force is a surface tension, which is given by $p_{T} \simeq \sigma / R \simeq 2 \sqrt{2 \lambda} \eta^{3} / 3 R$ (as a pressure). It smooths out the small scale structure on the wall and straightens the wall up to the horizon scale. The second force is a volume pressure, which is given by $p_{V} \simeq \Lambda=4 \epsilon \eta^{4} / 3$. It accelerates the wall against the false vacuum regions due to the energy difference between the two vacua and collapses the wall. The evolution of domain wall networks depends on when and which of the two forces dominate. The volume pressure dominates when $p_{V} \simeq p_{T}$ is satisfied, and the corresponding wall curvature radius is

$$
R \simeq \sqrt{\frac{\lambda}{2}}(\epsilon \eta)^{-1}
$$

If we require that the wall has been straighten up to the horizon scale before the volume pressure becomes effective, we obtain the condition $R>H^{-1}\left(t_{c}\right)$ where $R$ is given by Eq. (8) and $H\left(t_{c}\right)$ is the Hubble parameter at the phase transition $\left(T=T_{c}=2 \eta\right)$. This condition gives an upper bound for $\epsilon$ :

$$
\epsilon<4.7 \times\left(\lambda g_{*}\right)^{1 / 2} \frac{\eta}{M_{P}},
$$

where $g_{*}$ is the number of relativistic degrees of freedom at $T_{c}$ and $M_{P}$ is the Planck mass. On the other hand, a requirement that domain walls do not dominate the energy density of the universe gives a lower bound for $\epsilon$. When domain wall networks are in scaling regime, the energy density of domain walls is estimated as $\rho_{w} \simeq \sigma / H^{-1} \simeq$ $\sqrt{2 \lambda} \eta^{3} / 3 t$. If we assume the radiation dominated universe, the total energy density of the universe is given by $\rho \simeq 3 M_{P}^{2} / 32 \pi t^{2}$. The domain walls start to dominate the universe $\left(\rho_{w} / \rho \simeq 1\right)$ when $t=t_{W D}$, where

$$
t_{W D} \simeq \frac{9 M_{P}^{2}}{64 \pi} \sqrt{\frac{2}{\lambda}} \eta^{-3} .
$$

Meanwhile, the typical time scale of the collapse of the wall network is given by the condition $H^{-1}\left(t_{\mathrm{dec}}\right)=2 t_{\mathrm{dec}} \simeq R$ where $R$ is given by Eq. (8):

$$
t_{\mathrm{dec}} \simeq \frac{1}{2} \sqrt{\frac{\lambda}{2}}(\epsilon \eta)^{-1}
$$


Therefore, the condition that domain walls collapse before wall domination is $t_{\mathrm{dec}}<t_{W D}$, from which we obtain

$$
\epsilon>\frac{16 \pi}{9} \lambda\left(\frac{\eta}{M_{P}}\right)^{2}
$$

To sum up, the domains with infinite size appear if the condition given by Eq. (7) is satisfied, and the domain walls collapse before wall domination if $\epsilon$ satisfies Eqs. 9 and $(12$. The decay time of the domain walls is given by Eq. (11).

\section{CALCULATION OF GRAVITATIONAL WAVES}

In this section, we describe how to calculate the spectrum of the stochastic gravitational wave backgrounds produced by domain walls. We use the formalism of "Green's function method" developed by Dufaux et al. 40, 41. Originally this formalism was applied to the production of gravitational waves from preheating [42, 43] after inflation. However, this framework is applicable to general scalar fields in expanding universe, especially the Higgs field forming domain walls considered here. In the following, we summarize the results of 40 .

Assuming a spatially flat Friedmann-Robertson-Walker background, gravitational waves are represented by the spatial metric perturbation

$$
d s^{2}=a^{2}(\tau)\left[-d \tau^{2}+\left(\delta_{i j}+h_{i j}\right) d x^{i} d x^{j}\right]
$$

where $h_{i j}$ satisfies the condition $\partial_{i} h_{i j}=h_{i}^{i}=0$. The evolution of $h_{i j}$ is described by the linearized Einstein equation

$$
\ddot{h}_{i j}+3 H \dot{h}_{i j}-\frac{\nabla^{2}}{a^{2}} h_{i j}=16 \pi G T_{i j}^{T T}
$$

where $T_{i j}^{T T}$ is the transverse-traceless part of the stress-energy tensor, which is computed by applying the projection operator in the momentum space

$$
\begin{aligned}
T_{i j}^{T T}(\tau, \mathbf{k}) & =\Lambda_{i j, k l}(\hat{k}) T_{i j}(\tau, \mathbf{k})=\Lambda_{i j, k l}(\hat{k})\left\{\partial_{k} \phi \partial_{l} \phi\right\}(\tau, \mathbf{k}), \\
\Lambda_{i j, k l}(\hat{k}) & =P_{i k}(\hat{k}) P_{j l}(\hat{k})-\frac{1}{2} P_{i j}(\hat{k}) P_{k l}(\hat{k}), \\
P(\hat{k}) & =\delta_{i j}-\hat{k}_{i} \hat{k}_{j},
\end{aligned}
$$

where $\hat{k}=\mathbf{k} /|\mathbf{k}|$ and $\left\{\partial_{k} \phi \partial_{l} \phi\right\}(\tau, \mathbf{k})$ is the Fourier transform of $\partial_{k} \phi(\tau, \mathbf{x}) \partial_{l} \phi(\tau, \mathbf{x})$.

Suppose that the source term is nonzero during the interval $\tau_{i} \leq \tau \leq \tau_{f}$. In this case, the equation of motion (14) is solved by using the Green's function in $\tau_{i} \leq \tau \leq \tau_{f}$, and the solution can be matched to the source-free solution in $\tau \geq \tau_{f}$. In the radiation dominated universe, the result is 40]

$$
\bar{h}_{i j}(\tau, \mathbf{k})=A_{i j}(\mathbf{k}) \sin \left[k\left(\tau-\tau_{f}\right)\right]+B_{i j}(\mathbf{k}) \cos \left[k\left(\tau-\tau_{f}\right)\right] \quad \text { for } \quad \tau \geq \tau_{f},
$$

where

$$
\begin{aligned}
& A_{i j}(\mathbf{k})=\frac{16 \pi G}{k} \int_{\tau_{i}}^{\tau_{f}} d \tau^{\prime} \cos \left[k\left(\tau_{f}-\tau^{\prime}\right)\right] a\left(\tau^{\prime}\right) T_{i j}^{T T}\left(\tau^{\prime}, \mathbf{k}\right) \\
& B_{i j}(\mathbf{k})=\frac{16 \pi G}{k} \int_{\tau_{i}}^{\tau_{f}} d \tau^{\prime} \sin \left[k\left(\tau_{f}-\tau^{\prime}\right)\right] a\left(\tau^{\prime}\right) T_{i j}^{T T}\left(\tau^{\prime}, \mathbf{k}\right)
\end{aligned}
$$

and $\bar{h}_{i j}=a(\tau) h_{i j}$

The energy density of gravitational waves is given by the spatial average of the product of $\dot{h}_{i j}$ (see e.g. [19])

$$
\rho_{\mathrm{gw}}=\frac{1}{32 \pi G}\left\langle\dot{h}_{i j}(t, \mathbf{x}) \dot{h}_{i j}(t, \mathbf{x})\right\rangle=\frac{1}{32 \pi G a^{4}} \frac{1}{V} \int \frac{d^{3} k}{(2 \pi)^{3}} \bar{h}_{i j}^{\prime}(\tau, \mathbf{k}) \bar{h}_{i j}^{\prime *}(\tau, \mathbf{k}),
$$

where a prime denotes a derivative with respect to the conformal time $\tau$ and $V$ is the comoving volume. Strictly speaking, there are other contributions for the right hand side of Eq. 20p: one is cross terms between $\bar{h}_{i j}$ and $\bar{h}_{i j}^{\prime}$ which turn out to vanish under the reality condition of $\bar{h}_{i j}(\tau, \mathbf{x})$, and another is the term proportional to $H^{2}$ which is ignorable if we consider only the modes inside the horizon at present time $k / a_{0} \gg H_{0}$. Substituting Eq. (18) into 
Eq. (20) and taking the time average over a period of the oscillations caused by the sine and cosine terms in Eq. 18 . to eliminate these oscillations, we find

$$
\rho_{\mathrm{gw}}(t)=\frac{4 \pi G}{a^{4}(t) V} \int \frac{d^{3} k}{(2 \pi)^{3}} \sum_{i, j}\left\{\left|\int_{\tau_{i}}^{\tau_{f}} d \tau^{\prime} \cos \left(k \tau^{\prime}\right) a\left(\tau^{\prime}\right) T_{i j}^{T T}\left(\tau^{\prime}, \mathbf{k}\right)\right|^{2}+\left|\int_{\tau_{i}}^{\tau_{f}} d \tau^{\prime} \sin \left(k \tau^{\prime}\right) a\left(\tau^{\prime}\right) T_{i j}^{T T}\left(\tau^{\prime}, \mathbf{k}\right)\right|^{2}\right\} .
$$

The present spectrum is represented by the fraction of energy density against the total energy density of the universe per logarithmic frequency interval

$$
\Omega_{\mathrm{gw}} h^{2}=\frac{h^{2}}{\rho_{c, 0}} \frac{d \rho_{\mathrm{gw}}\left(t_{0}, f\right)}{d \ln f}=\frac{h^{2}}{\rho_{c, 0}} \frac{d \rho_{\mathrm{gw}}\left(t_{0}, k\right)}{d \ln k},
$$

where $f$ is the frequency of gravitational waves, $\rho_{c, 0}$ is the critical energy density today, and $h$ is the renormalized Hubble parameter: $H_{0}=100 \mathrm{kmsec}^{-1} \mathrm{Mpc}^{-1}$. Taking account of the fact that energy density of gravitational waves redshifts as $\rho_{\mathrm{gw}} \propto a^{-4}$, and from Eqs. 21 and 22 , we obtain

$$
\Omega_{\mathrm{gw}} h^{2}=\left(\frac{g_{0}}{g_{*}}\right)^{1 / 3} \frac{\Omega_{R} h^{2}}{\rho_{R}\left(t_{i}\right)} \frac{G k^{3}}{2 \pi^{2} V} I_{k}
$$

where

$$
I_{k}=\int d \Omega_{k} \sum_{i, j}\left\{\left|\int_{\tau_{i}}^{\tau_{f}} d \tau^{\prime} \cos \left(k \tau^{\prime}\right) a\left(\tau^{\prime}\right) T_{i j}^{T T}\left(\tau^{\prime}, \mathbf{k}\right)\right|^{2}+\left|\int_{\tau_{i}}^{\tau_{f}} d \tau^{\prime} \sin \left(k \tau^{\prime}\right) a\left(\tau^{\prime}\right) T_{i j}^{T T}\left(\tau^{\prime}, \mathbf{k}\right)\right|^{2}\right\}
$$

Here $g_{0}=3.36$ is the number of radiation degrees of freedom today, $\Omega_{R} h^{2}=4.15 \times 10^{-5}$ is the ratio of the radiation energy density to the critical energy density today, and $\rho_{R}\left(t_{i}\right)$ is the radiation energy density at the initial time. $\Omega_{k}$ is a unit vector representing the direction of $\mathbf{k}$ and $d \Omega_{k}=d \cos \theta d \phi$. We set the scale factor at the initial time as $a\left(t_{i}\right)=1$. Meanwhile, the frequency observed today is given by the comoving momentum redshifted by $a\left(t_{0}\right)^{-1}$

$$
f=\frac{k}{2 \pi a\left(t_{0}\right)}=\frac{k}{2 \pi}\left(\frac{g_{0}}{g_{*}}\right)^{1 / 3} \frac{T_{0}}{T_{i}}
$$

where $T_{0}=2.725 \mathrm{~K}$ is the temperature of the universe observed today and $T_{i}$ is the initial temperature (to be specified later).

The computational strategy is as follows: first, we obtain the time evolution of scalar fields in the real space $\phi(t, \mathbf{x})$ by the lattice simulation, then we compute the TT projected stress-energy tensor as Eqs. (15)-(17), and finally we increment the time integral in Eq. (24) to obtain the spectrum by using Eqs. (23) and (25).

\section{NUMERICAL SIMULATION}

\section{A. Problem in Numerical Study and Choice of Parameters}

The evolution equation of domain walls is highly nonlinear and we are forced to use numerical analysis. However, there is a technical problem in computing the evolution of domain walls numerically: one must consider two extremely different length scales simultaneously. One is the width of the wall $\delta_{w} \sim \eta^{-1}$, which must be bigger than a lattice spacing throughout the numerical simulation in order to resolve domain walls by individual unit of lattice. But if we perform simulations in the comoving box, the physical lattice spacing expands as $\propto a(t)$, and becomes unable to resolve the wall in the finite simulation time scale. The other length scale is the Hubble horizon, which must be smaller than the simulation box in order to avoid unphysical effect caused by the finite size of the simulation box. Unfortunately, there is a large gap between these two length scales by a factor of the ratio between the mass scale of the scalar field and the Planck scale as

$$
\delta_{w} \sim \eta^{-1} \ll\left(\frac{M_{P}}{\eta}\right) \eta^{-1} \sim H^{-1} .
$$

Therefore, we can not simulate the physical evolution of domain walls for a long time for energy scale $\eta$ sufficiently smaller than the Planck scale. 
The standard PRS algorithm 28 avoids this difficulty described above by keeping the comoving wall width constant in time, to maintain the width resolvable throughout a simulation. However, in this work we can not apply this method, since the energy of gravitational waves radiated by domain walls is estimated incorrectly due to the unphysical nature of the wall width.

Hence we directly solve the physical equation of motion

$$
\ddot{\phi}+3 H \dot{\phi}-\frac{\nabla^{2}}{a^{2}} \phi+\frac{d V}{d \phi}=0,
$$

with the potential given by Eq. (1). This restricts the time scale of the simulation and the model parameters. We must choose $\eta$ close to the Planck scale due to the fact represented by Eq. 267, but if we choose $\eta$ close enough to $M_{P}$, the onset of scaling is delayed in the unit of Hubble time (we choose time coordinate normalized by the initial Hubble time $t_{i} \sim H^{-1}\left(t_{i}\right)$, see Appendix A 1) due to the degeneracy between the inverse mass scale $\eta^{-1}$ and the Hubble radius $H^{-1}$. As the marginal possible parameters, we choose $\lambda=1.0$ and $\eta=1.05 \times 10^{17} \mathrm{GeV}$. We assume the radiation dominated era and the relativistic degree of freedom is constant given by $g_{*}=100$ during the simulation. The final time is set to be $t_{f}=151 t_{i}$ where $t_{i}$ is initial time chosen so that the initial temperature is twice of the critical temperature of the phase transition $\left(T_{i}=2 T_{c}=4 \eta, t_{i}=t_{c} / 4\right)$. We performed lattice simulations with $256^{3}$ points in the cases with $b=25$ and $b=50$, where $b$ is the (comoving) box size in the unit of $t_{i}$. In these parameters, the resolution of the wall width is well maintained and the horizon scale is smaller than the simulation box even at the final time (see Appendix A 1).

For biased domain walls, we choose the parameter $\epsilon$ such that the conditions Eqs. (9) and (12) are satisfied:

$$
\frac{16 \pi}{9} \lambda\left(\frac{\eta}{M_{P}}\right)^{2}<\epsilon<4.7 \times\left(\lambda g_{*}\right)^{1 / 2} \frac{\eta}{M_{P}} .
$$

In the parameters described above, Eq. 28 corresponds to the condition $4.1 \times 10^{-4}<\epsilon<0.40$. We run the simulations varying $\epsilon$ in the range $\epsilon=0.015,0.02,0.025,0.03,0.035$, and 0.04 which satisfy the condition Eq. (28). These values also satisfy the condition for an appearance of the infinite size of domains, Eq. (7).

We emphasize that the dynamical range of the simulations is rather small compared with realistic cosmological dynamical ranges. The actual dynamical range is 12 in conformal time. For the domain wall evolution, this is reduced to less than $\left(151 t_{i} / 30 t_{i}\right)^{1 / 2} \simeq 2.2$ since the result with $\epsilon=0$ do not approach scaling until $t=30 t_{i}$ (see next subsection and Fig. 2). On the other hand, the typical time scale for the wall domination is 64 [according to Eq. (10)]. It is extremely difficult to improve dynamical ranges without using PRS algorithm. Future simulations with much larger dynamical ranges should confirm and improve the results of our current simulations.

\section{B. Evolution of Domain Wall Networks}

The typical evolution of stable $(\epsilon=0)$ and biased $(\epsilon \neq 0)$ domain wall networks is shown in Fig. 1. The initial thermal fluctuations of the scalar field are damped around $t=30 t_{i}$, and domain walls extended over the horizon scale are formed. In the case $\epsilon=0$, the wall networks evolve with annihilation and reconnection to maintain scaling property. If we turn on the bias, instead, the region occupied by false vacuum after the phase transition is smaller than the unbiased case, and walls gradually collapse due to the volume pressure.

To see more quantitatively, we calculate time evolution of the comoving area density of domain walls as shown in Fig. 2 (see Appendix A3 for a detail of the calculation). From this figure we can see that the scaling regime in which the area density scales as $t^{-1 / 2}$ begins around $t=30 t_{i}$ for the case $\epsilon=0$. In the $b=25$ case, the scaling law breaks down around the late stage of the evolution. This may be caused by an unphysical effect of the finiteness of the simulation box, that is, the horizon scale become comparable to the size of the simulation box around the final time (the similar deviation from the scaling solution can be found in [28, 33, 34]).

In the primary thermal stage $t \lesssim 30 t_{i}$, the correlation length of the scalar field is determined by mass scale of the scalar field $\xi_{\text {corr }} \sim m^{-1} \sim \eta^{-1}$ rather than the Hubble horizon scale. In this stage, the value of the scalar field changes randomly in the short length scale given by $\xi_{\text {corr }}$, thus $A / V$ remains nearly constant. Note that if $b t_{i}>\xi_{\text {corr }} \sim m^{-1}$, by increasing box size $b$ with the fixed number of grids one can count more points where the scalar field change their sign, leading to increase the value of $A / V$. In fact, the value of $A / V$ at the primary stage $t \lesssim 30 t_{i}$ in the case $b=50$ is larger than that in the case $b=25$, as shown in Fig. 2 .

As we turn on the bias, the value of $A / V$ falls by a factor of $\mathcal{O}(10-100)$ in the simulation time scale. The typical time scale of the decay of the area density is well described by the estimation Eq. (11) except a factor of $\mathcal{O}(1)$. There are oscillations of $A / V$ after the decay of domain walls. This can be interpreted as the radiation of the energy stored in walls as the scalar field oscillating around the true vacuum [35. The difference in the amplitude of oscillations 

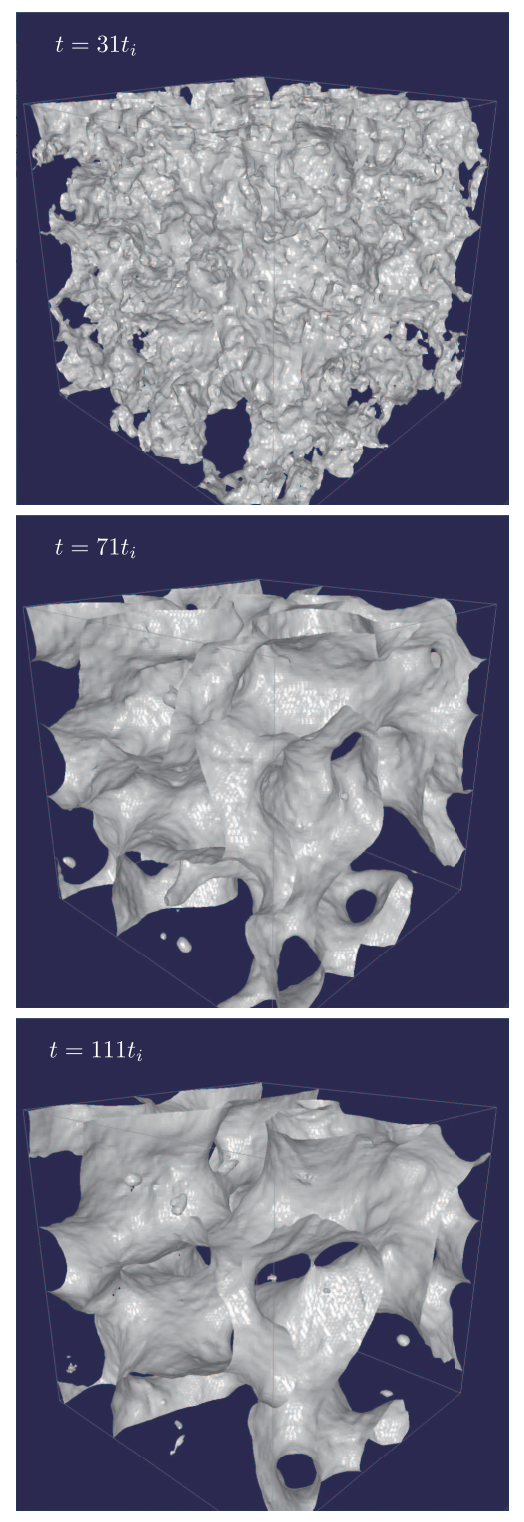
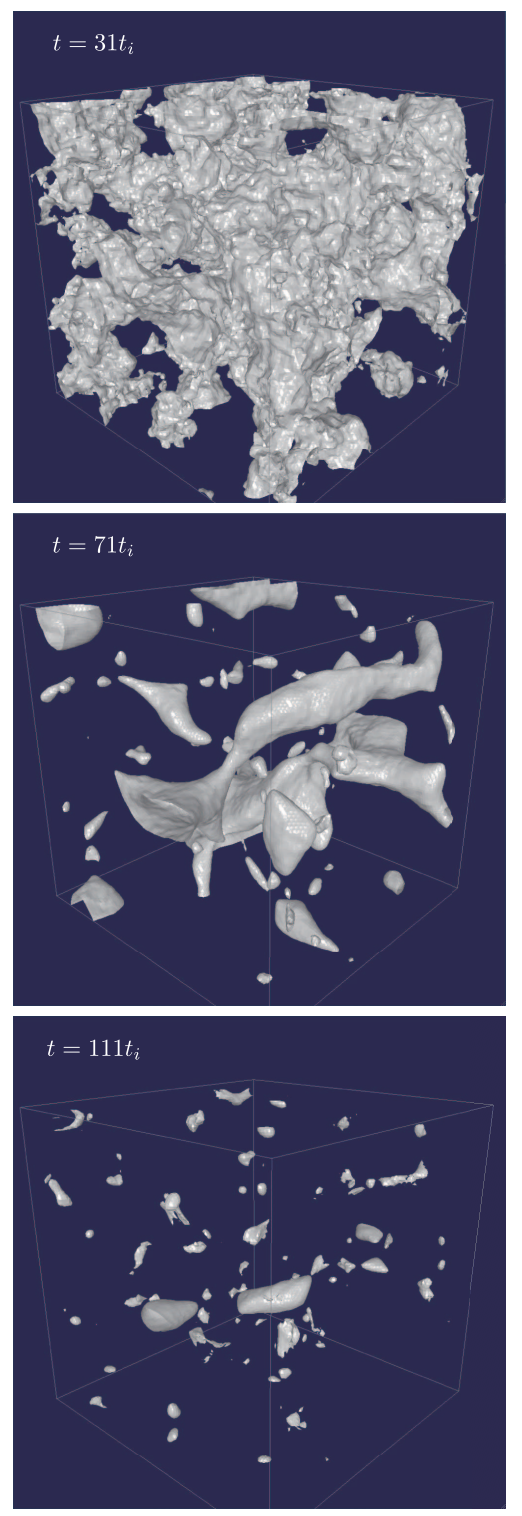

FIG. 1: The evolution of stable $(\epsilon=0$ : left $)$ and unstable $(\epsilon=0.02$ : right $)$ domain walls. The white surface corresponds to the region where the value of $\phi$ crosses the zero.

between the case with $b=25$ and $b=50$ may be due to the poor resolution in the simulation with $b=50$. If the wavelength of the scalar field radiations produced by the decay of domain walls is comparable or smaller than the small scale cutoff $\left(\sim \eta^{-1}\right)$, the typical length scale over which the scalar field varies becomes shorter than the lattice spacing. This makes the value of $A / V$ inaccurate because we estimate the value $A / V$ as a sum of the link of points where $\phi$ has different signs (see Appendix A 3). In the case with $b=50$, the lattice spacing $\delta x$ is larger than that with $b=25$ and it is likely to lose many links over which the scalar field changes its signs. Therefore, the oscillation of the $A / V$ in the case with $b=50$ is less violent than that with $b=25$, as we see in Fig. 2 .

\section{Spectrum of Gravitational Waves}

By using the method described in Section III, we compute the spectrum of gravitational waves produced by domain walls. The results are shown in Fig. 3. To take small $b$ with fixed number of lattice points corresponds to increasing the spatial resolution. Therefore, the frequency range computed in the case with $b=25$ is higher than that in the case with $b=50$. The two figures shown in Fig. 3 seem to be different in spite of the fact that the two results are obtained 

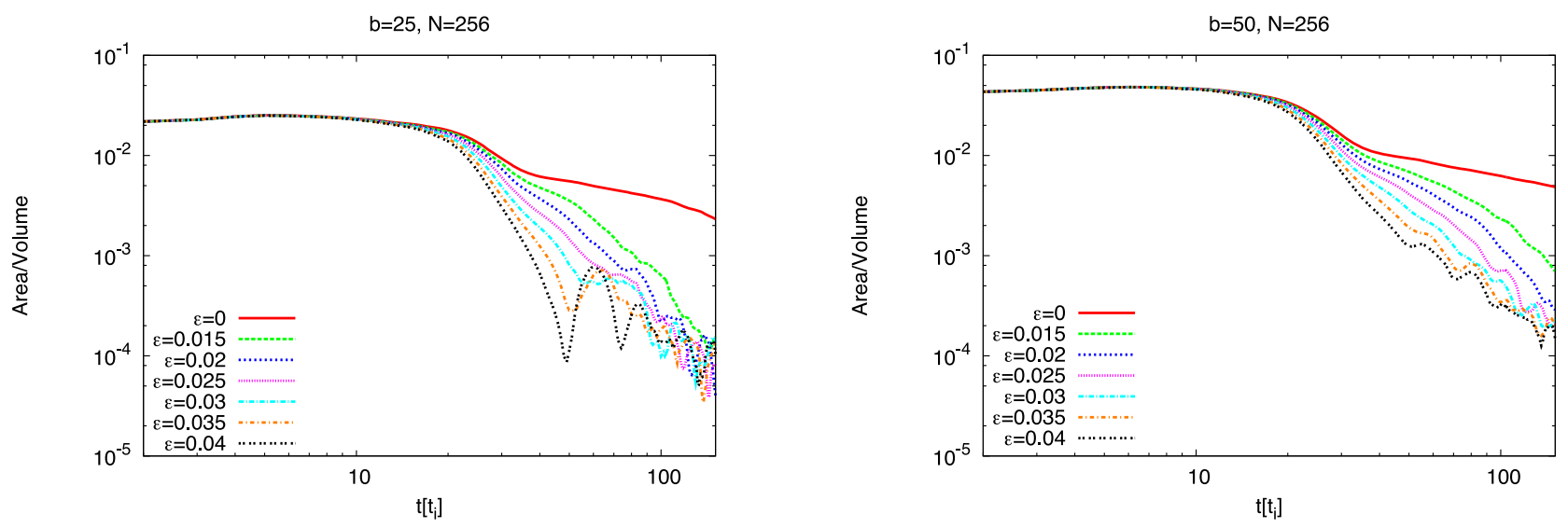

FIG. 2: The time evolution of the comoving area density of domain walls for various values of $\epsilon$. The left panel shows the result in the case with the box size $b=25$ and the right panel shows that in the case with $b=50$.

from calculations with completely the same parameters except $b$. For example, there is a peak in high frequency edge around $2 \times 10^{11} \mathrm{~Hz}$ of the spectrum with $b=50$, but the corresponding peak is not found in the spectrum with $b=25$. Hence this high frequency peak in $b=50$ is thought to be unphysical noise due to the poor resolution for a small scale. Another difference is that the low frequency tail of the spectrum in $b=25$ is steeper than that in $b=50$. This is due to the finite volume effect which promotes walls to collapse around the final time in $b=25$, as shown in Fig. 2. Because of the limitation in our computer memory, we can not perform the simulation with maintaining physical resolution over full range of gravitational wave spectra. From now on, therefore, we use the result of the case with $b=50$ and ignore the high frequency peak around $2 \times 10^{11} \mathrm{~Hz}$.
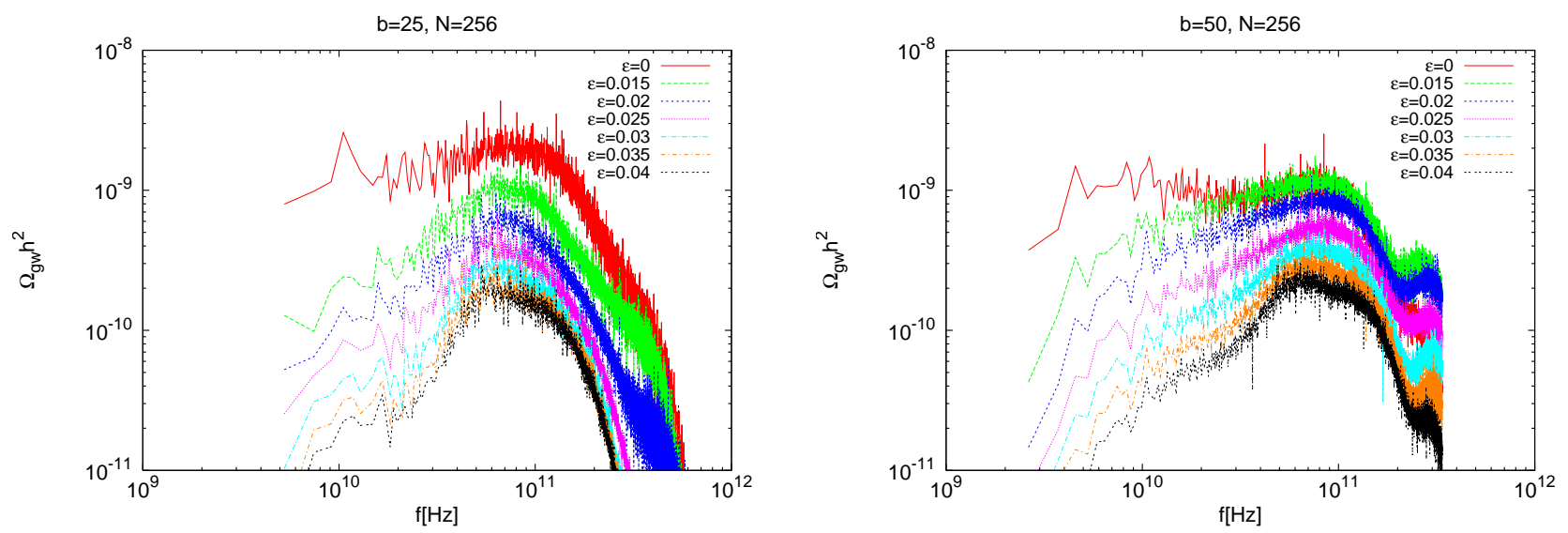

FIG. 3: The spectrum of gravitational waves from domain walls for various values of $\epsilon$. The left panel shows the result in the case with the box size $b=25$ and the right panel shows that in the case with $b=50$.

In Fig. 4. we show the time evolution of the gravitational wave spectra for several values of $\epsilon$ in the case $b=50$. There are also the unphysical high frequency peak in those spectra, but we ignore them for the reason described above. By comparing Fig. 2 and Fig. 4 we can see that the spectrum of gravitational waves evolves correspondingly to the evolution of the scalar field. In the primary stage $\left(t \lesssim 30 t_{i}\right)$, the thermally distributed scalar field produces the spectrum which has a peak around the frequency $f \sim 10^{11} \mathrm{~Hz}$, and after the formation of scaling wall networks $\left(t \gtrsim 50 t_{i}\right)$ there is an increase in gravitational wave amplitudes of low frequency modes. These low frequency modes turn out to correspond the horizon scale at the time when gravitational waves are produced. We will investigate it in detail in Section $\mathrm{V}$. In the late stage, if $\epsilon=0$, the amplitudes of the gravitational waves keep growing in both 
high frequency of the small scale and low frequency of the horizon scale. On the other hand, if $\epsilon \neq 0$, the growth in low frequency terminates when walls begin to collapse, and only high frequency modes grow in the late stage. The resulting spectrum has the low frequency edge and the high frequency peak, and gradually increases in intermediate modes.
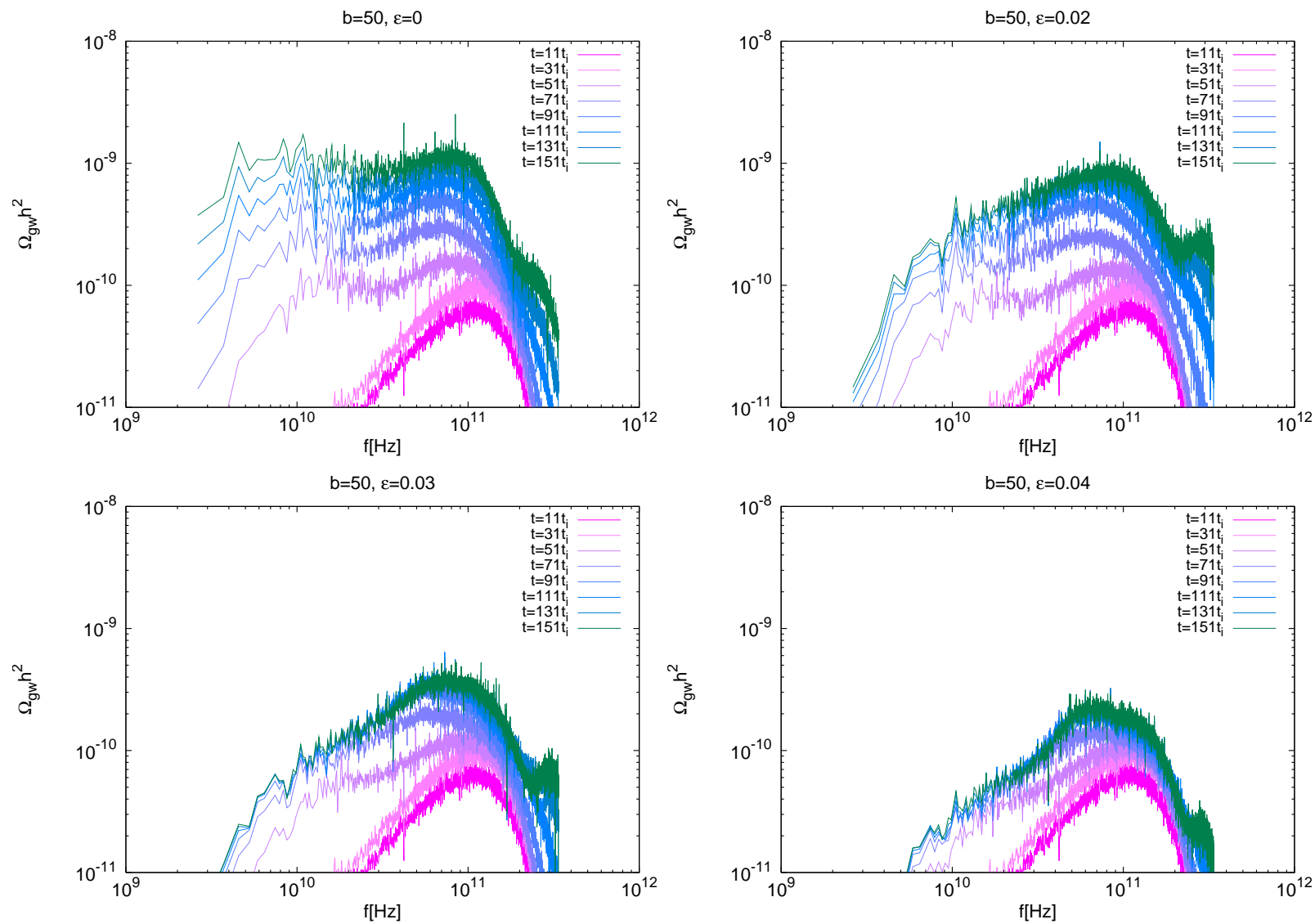

FIG. 4: The time evolution of the gravitational wave spectra for various values of $\epsilon$ (in the case $b=50$ ). The spectra are shown from the time $t=11 t_{i}$ (pink) to $t=151 t_{i}$ (green) with the interval $\Delta t=20 t_{i}$.

\section{ANALYTIC ESTIMATION AND PERSPECTIVE FOR OBSERVATIONS}

In this section we discuss the physical interpretation of the numerical results. We check whether the results of the numerical simulation match the naive analytic estimations. Finally we extrapolate the results into the general parameter space and estimate the amplitude and the frequency of gravitational waves observed today for various parameters. In particular we discuss whether the spectrum is observable in the future gravitational wave experiments.

\section{A. Physical Interpretation of the Spectrum}

There are two mechanisms relevant for production of gravitational waves from domain wall dynamics. One is due to the relativistic motion of walls in the universe, and another is due to the interactions of domain wall networks such as collision, separation, and reconnection of walls. Considering the scaling property of domain walls, the typical scale relevant for the former is the horizon scale at the time when gravitational waves are produced. Meanwhile, the typical scale relevant for the latter is the small scale of the structure on the domain wall. As long as domain walls are in the scaling regime, both of these two mechanisms are efficient, and the resulting spectrum extends over a broad frequency band between the low frequency of the horizon scale and the high frequency of the small scale structure of 
the wall, as shown in Fig. 3. Instead, the growth of the amplitude in low frequency modes terminates when domain walls begin to collapse if there is a bias.

From the above considerations, we conjecture that the form of the spectra is determined as follows. There is a peak in the high frequency corresponding to the small scale structure of the wall (i.e. the wall width). The spectrum gradually decreases toward the low frequency, and there is an 'edge' in the low frequency corresponding to the horizon scale. For causality reasons, there are no modes beyond the horizon scale.

In the following, we estimate the amplitude and the frequency of gravitational waves analytically in order to justify the above considerations.

\section{B. Analytic Estimation}

First we estimate the peak frequency. The peak is given by the interaction of domain wall networks and we naively estimate that the peak frequency corresponds to the width of the domain wall. One can think of this process as the collision of wave packets of classical scalar fields whose wavelength is about $\lambda_{\text {peak }} \sim \delta_{w} \sim\left(\lambda^{1 / 2} \eta\right)^{-1}$. Then, the physical wave number of the peak of gravitational waves at the time $t_{*}$ when they are produced is $k_{\text {peak }} / a\left(t_{*}\right)=$ $2 \pi / \lambda_{\text {peak }}=2 \pi \lambda^{1 / 2} \eta$, where $k_{\text {peak }}$ is the comoving wave number corresponding to the peak frequency. Taking redshift into account, the peak frequency observed today is obtained as

$$
\begin{aligned}
f_{\text {peak }} & =\frac{k_{\text {peak }}}{2 \pi a\left(t_{0}\right)}=\left(\frac{a\left(t_{*}\right)}{a\left(t_{0}\right)}\right) \lambda^{1 / 2} \eta \\
& =3.5 \times 10^{11} \times \lambda^{1 / 2}\left(\frac{100}{g_{*}}\right)^{1 / 3}\left(\frac{t_{*}}{151 t_{i}}\right)^{1 / 2} \mathrm{~Hz} .
\end{aligned}
$$

Setting $t_{*}$ to the final time of the simulation, $151 t_{i}$, the estimation of the peak frequency gives $3.5 \times 10^{11} \mathrm{~Hz}$, which agrees with our numerical results except the factor of $\mathcal{O}(1)$. Note that if we assume the entropy conservation, the dependence $k_{\text {peak }} \propto \eta$ is canceled by the factor $a\left(t_{i}\right) / a\left(t_{0}\right) \propto 1 / \eta$. Therefore, generically the peak frequency is located in the high frequency of order $10^{11} \mathrm{~Hz}$ regardless of the value of $\eta$.

Next, we estimate the amplitude of gravitational waves observed today. The energy of gravitational waves produced by domain walls is estimated as $E_{\mathrm{gw}} \sim G M_{D W}^{2} / R_{*}$, where $R_{*}$ is a typical length scale over which the energy distributes and $M_{D W}$ is the mass scale of the domain wall. We do not specify the value of $R_{*}$ since the result is not depend on $R_{*}$ as shown shortly. We estimate $M_{D W} \sim \sigma R_{*}^{2}=\sqrt{2 \lambda} \eta^{3} R_{*}^{2} / 3$, and the energy density of gravitational waves at the production time is $\rho_{\mathrm{gw}}\left(t_{*}\right) \sim E_{\mathrm{gw}} / R_{*}^{3} \sim(8 \lambda / 9)\left(\eta / M_{P}\right)^{2} \eta^{4}$. By redshifting it, we find the amplitude of the gravitational waves observed today as

$$
\Omega_{\mathrm{gw}} h^{2} \sim\left(\frac{a\left(t_{*}\right)}{a\left(t_{0}\right)}\right)^{4} \frac{\rho_{\mathrm{gw}}\left(t_{*}\right) h^{2}}{\rho_{c, 0}} \sim 7.5 \times 10^{-10} \times \lambda\left(\frac{100}{g_{*}}\right)^{4 / 3}\left(\frac{\eta}{1.05 \times 10^{17} \mathrm{GeV}}\right)^{2}\left(\frac{t_{*}}{151 t_{i}}\right)^{2},
$$

which agrees with the peak amplitude $\Omega_{\mathrm{gw}} h^{2} \sim \mathcal{O}\left(10^{-9}\right)$ of the numerical results.

We also estimate the location of the low frequency edge in the spectrum which corresponds to the horizon scale. The physical wave number corresponding to the horizon scale at time $t$ is given by $k_{\text {hor }} / a(t)=2 \pi H(t)=\pi / t$. Hence the frequency observed today is given by

$$
f_{\text {hor }}=\frac{k_{\text {hor }}}{2 \pi a\left(t_{0}\right)}=\left(\frac{a(t)}{a\left(t_{0}\right)}\right) \frac{1}{2 t}=6.58 \times 10^{10} \times\left(t / t_{i}\right)^{-1 / 2}\left(\frac{\eta}{1.05 \times 10^{17} \mathrm{GeV}}\right) \mathrm{Hz} .
$$

Notice that $t_{i} \propto \eta^{-2}$ and hence $f_{\text {hor }}$ is independent of both $\eta$ and $t_{i}$ as it should be. For example, the low frequency edge at the final time of the simulation $t=151 t_{i}$ becomes $f_{\text {hor }} \simeq 5 \times 10^{9} \mathrm{~Hz}$, which reproduces the location of the edge in the spectrum obtained by numerical simulations.

Before moving to the next subsection, we discuss the origin of the spectrum produced during the primary stage $\left(t \lesssim 30 t_{i}\right)$ of the scalar field evolution. In this stage, the scalar field fluctuates randomly with correlation length roughly given by inverse of the temperature $\xi_{\text {corr }} \sim T^{-1}$, and gravitational waves can be produced via the quadratic interaction of the scalar field. We denote the amplitude of the scalar field fluctuations by $\delta \phi$. From the equation of motion for the metric perturbation, Eq. (14), a typical amplitude of gravitational wave $h_{g}$ with the comoving wave number $k$ is estimated as $k^{2} h_{g} \sim 16 \pi \nabla \phi \nabla \phi / M_{P}^{2} \sim 16 \pi k^{2} \delta \phi^{2} / M_{P}^{2}$, thus we obtain $h_{g} \sim 50 \times \delta \phi^{2} / M_{P}^{2}$. On the other hand, the ratio between the energy density of gravitational waves and the total energy density of the universe at the production time is estimated as $\left(\Omega_{\mathrm{gw}}\right)_{*} \sim \rho_{\mathrm{gw}} / \rho_{R}\left(t_{*}\right) \sim \dot{h}_{g}^{2} / G \rho_{R}\left(t_{*}\right) \sim \dot{h}_{g}^{2} / H^{2}\left(t_{*}\right) \sim t_{*}^{2} \dot{h}_{g}^{2} \sim h_{g}^{2}$, where $\rho_{R}\left(t_{*}\right)$ is the energy density of the radiation at $t=t_{*}$ and we assume the radiation dominated universe. In our simulations, the scalar 
field initially fluctuates with amplitude $\delta \phi \sim T_{i} \sim \eta \sim 10^{17} \mathrm{GeV}$, which gives $\left(\Omega_{\mathrm{gw}}\right)_{*} \sim h_{g}^{2} \sim 10^{3} \times\left(\delta \phi / M_{P}\right)^{4} \sim 10^{-5}$. The amplitude of these gravitational waves observed today is [16]

$$
\Omega_{\mathrm{gw}} h^{2}=1.67 \times 10^{-5}\left(\frac{100}{g_{*}}\right)^{1 / 3}\left(\Omega_{\mathrm{gw}}\right)_{*} \sim 10^{-10} .
$$

This agrees with our numerical results (see the spectra of $t=11 t_{i}$ and $31 t_{i}$ in Fig. 4p. The location of the peak (i.e. $f \sim 10^{11} \mathrm{~Hz}$ ) is almost unchanged from the early time to the final time. This is because the typical frequency of gravitational waves produced in the early time is roughly given by correlation length of the scalar field which is determined by the mass scale $m \sim \sqrt{3 \lambda} \eta$, and this scale is nearly equal to the typical scale of the small structure on the domain wall $\sim \lambda^{1 / 2} \eta$.

\section{Fitting the Numerical Results}

The numerical results reveal that the gravitational wave spectra appear in rather high frequency band. This is partially due to the fact that we assume very high energy scale, $\eta \simeq 10^{17} \mathrm{GeV}$. For the case with lower $\eta$, it is impossible to perform the numerical computation correctly because of the technical restriction described in Section IVA. Instead, we assume the parameter dependence of analytic estimations obtained above, and fit the numerical factor into the results of numerical simulations to derive the formulae for the frequency and the amplitude of peak and edge in the gravitational wave spectra. In deriving these formulae, we check the $\epsilon$ dependence of the numerical results. The difference in the bias $\epsilon$ affects the time scale of the collapse of the domain wall networks [see Eq. (11)]. In the following we assume that the typical time of the production of gravitational waves $t_{*}$ is identical to the decay time of the domain walls $t_{\mathrm{dec}}$, and estimate $\epsilon$ dependence of the spectrum. Then we see if our numerical results follow the dependence.

Let us consider the peak frequency. Assuming the parameter dependence of Eq. 290 and replacing the numerical coefficient of $\mathcal{O}(1)$ by that of the numerical result, we obtain

$$
\begin{aligned}
f_{\text {peak }} & \simeq 9 \times 10^{10} \times \lambda^{1 / 2}\left(\frac{100}{g_{*}}\right)^{1 / 3}\left(\frac{t_{*}}{151 t_{i}}\right)^{1 / 2} \mathrm{~Hz} \\
& \simeq 9 \times 10^{9} \times \epsilon^{-1 / 2} \lambda^{3 / 4}\left(\frac{100}{g_{*}}\right)^{1 / 12}\left(\frac{\eta}{10^{17} \mathrm{GeV}}\right)^{1 / 2} \mathrm{~Hz} .
\end{aligned}
$$

In the second line, we substitute $t_{\text {dec }}$ given by Eq. 111) into $t_{*}$. The difference in $g_{*}$ dependence between the first line and the second line comes from $t_{i}=\left(45 M_{P}^{2} / 16 \pi^{2} g_{*} T_{i}^{4}\right)^{1 / 2} \propto g_{*}^{-1 / 2} \eta^{-2}$ which is determined by the Friedmann equation at the initial time. From Eq. (33), we expect that the location of the peak shifts to lower frequency for a larger value of $\epsilon$. This is because if $\epsilon$ is large, domain walls collapse in the early stage of their evolution, and the gravitational waves redshifts for a longer time than the case of a small $\epsilon$. In fact, the peak in our numerical simulations shifts to the lower frequency for a larger value of $\epsilon$ (see Fig. 3). But if $\epsilon$ is sufficiently large, the peak mixes with the primary spectrum produced at $t \lesssim 30 t_{i}$, therefore it is difficult to check precisely the $\epsilon$ dependence described by Eq. (33).

Next we consider the peak amplitude of the gravitational waves. Substituting $t_{*} \simeq t_{\mathrm{dec}}$ into Eq. (30), we obtain the parameter dependence of the amplitude $\Omega_{\mathrm{gw}} h^{2} \propto g_{*}^{-4 / 3} \lambda \eta^{2}\left(t_{\mathrm{dec}} / t_{i}\right)^{2} \propto g_{*}^{-1 / 3} \lambda^{2} \eta^{4} \epsilon^{-2}$. So the amplitude is proportional to $\epsilon^{-2}$. In Fig. 5. we plot the $\epsilon$ dependence of the peak amplitudes obtained by numerical results for $b=50$, and we found that the property $\Omega_{\mathrm{gw}} h^{2} \propto \epsilon^{-2}$ is indeed observed in our numerical results. By fitting the numerical coefficients into the line in Fig. 5, we obtain the peak amplitude

$$
\left(\Omega_{\mathrm{gw}} h^{2}\right)_{\text {peak }} \simeq 3 \times 10^{-13} \times \epsilon^{-2} \lambda^{-2}\left(\frac{100}{g_{*}}\right)^{1 / 3}\left(\frac{\eta}{10^{17} \mathrm{GeV}}\right)^{4} .
$$

Note that the result with $\epsilon=0.015$ slightly deviates from the line of $\epsilon^{-2}$ in Fig. 5 . There are some possibilities to explain this deviation. One explanation is that the deviation is due to the lack of the simulation time. If $\epsilon$ is sufficiently small, the domain walls have not completely collapsed in the simulation time. Therefore, there would be growth of $\Omega_{\mathrm{gw}} h^{2}$ if we run the simulation for a longer time. Another possibility is that the relation $\Omega_{\mathrm{gw}} h^{2} \propto \epsilon^{-2}$ is not exactly satisfied for small $\epsilon$. In fact, if we take the limit $\epsilon \rightarrow 0, \Omega_{\mathrm{gw}} h^{2}$ diverges. Moreover, we must be careful about the contribution of the energy density of domain walls to the total energy density of the universe for the case with small $\epsilon$, which is ignored here. Unfortunately, we can not test these possibilities in our current capability of computers. 


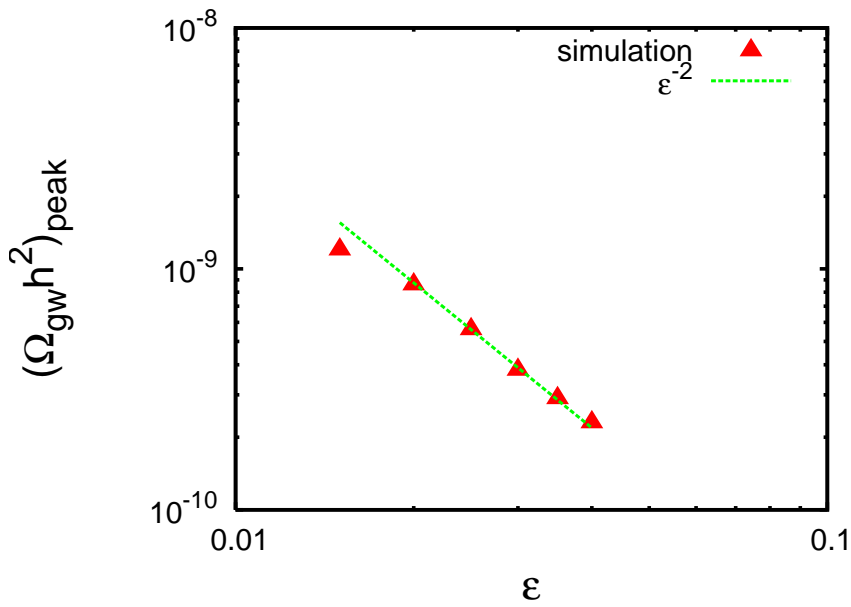

FIG. 5: The relation between $\left(\Omega_{\mathrm{gw}} h^{2}\right)_{\text {peak }}$ and $\epsilon$ obtained from the numerical results. The dotted line represents the fitting formula given in Eq. (34).

Now, we consider the intermediate frequency range between the high frequency peak and the low frequency edge. We parameterize the form of the spectrum in this range by $\Omega_{\mathrm{gw}} h^{2} \propto f^{n}$. In Fig. 66, we plot the value of $n$ from numerical results in the case with the box size $b=50$ for $\epsilon=0.015,0.02,0.025$, and 0.03 . We also include the data with $\epsilon=0$ for the power law fitting in Fig. 6. The result of $\epsilon=0$ is not the spectrum which we would observe because the walls do not annihilate in the simulation time. However, we expect that the form of the spectrum with $\epsilon \ll 1$ is well approximated by the result with $\epsilon=0$ if we assume that the low frequency modes of the gravitational waves are generated by the scaling evolution of the wall networks. Note that we omit the data for $\epsilon=0.035$ and 0.04 since the walls collapse without following scaling phase sufficiently and the form of the spectrum significantly different from that of the small value of $\epsilon$. By fitting the numerical results, we obtain the frequency dependence as

$$
\Omega_{\mathrm{gw}} h^{2} \propto f^{20 \epsilon+0.08} \quad \text { for } \quad f_{\text {edge }}<f<f_{\text {peak }} .
$$

For a large value of $\epsilon$, the domain walls immediately collapse after the formation, and there is little growth of the amplitude in the lower frequency modes corresponding to the horizon scale, resulting in a steeper spectrum. On the other hand, for a small value of $\epsilon$, domain walls are straightened up to horizon scale before they collapse. In this case, the difference in the value of $\Omega_{\mathrm{gw}} h^{2}$ between the peak and the edge is determined by the duration time of the collapse, which might be independent of $\epsilon$. Therefore, the value of the spectral index is independent of bias for $\epsilon \ll 1$ and then $n \simeq 0.08$. We emphasize that there might be large uncertainties in the parameterization in Eq. (35). If we write the frequency dependence as $\Omega_{\mathrm{gw}} h^{2} \propto f^{\alpha \epsilon+\beta}$, the standard errors for the coefficients $\alpha$ and $\beta$ are about $10 \%$ and $50 \%$, respectively. These errors arise from the fact that there are only a few data points because of the lack of the dynamical range of the simulations. It will cause large uncertainties in the magnitude of gravitational waves relevant to the observations when we extrapolate the result in Section $V D$.

Before closing this subsection, we consider the edge in low frequency. We can not read the precise location of the edge from the numerical results because of the large statistical variance in the low frequency modes. Therefore, here we just derive the parameter dependence of the frequency and the amplitude for the low frequency edge analytically, instead of fitting numerical coefficients into the results of the numerical simulations as the previous discussions. Substituting $t=t_{*} \simeq t_{\mathrm{dec}}$ into Eq. (31), we find

$$
f_{\text {edge }}=f_{\text {hor }}\left(t_{\mathrm{dec}}\right)=5 \times 10^{10} \times \epsilon^{1 / 2} \lambda^{-1 / 4}\left(\frac{100}{g_{*}}\right)^{1 / 12}\left(\frac{\eta}{10^{17} \mathrm{GeV}}\right)^{1 / 2} \mathrm{~Hz} .
$$

For small $\epsilon$, the life time of domain walls becomes long, and the horizon scale at $t_{\text {dec }}$ becomes large. Then $f_{\text {edge }}$ shifts to the lower frequency, as shown in $b=50$ case of Fig. 3 .

Also, by using Eq. (35), we can estimate the amplitude of gravitational waves at the edge as

$$
\left(\Omega_{\mathrm{gw}} h^{2}\right)_{\text {edge }} \simeq\left(\frac{f_{\text {edge }}}{f_{\text {peak }}}\right)^{20 \epsilon+0.08}\left(\Omega_{\mathrm{gw}} h^{2}\right)_{\text {peak }} .
$$




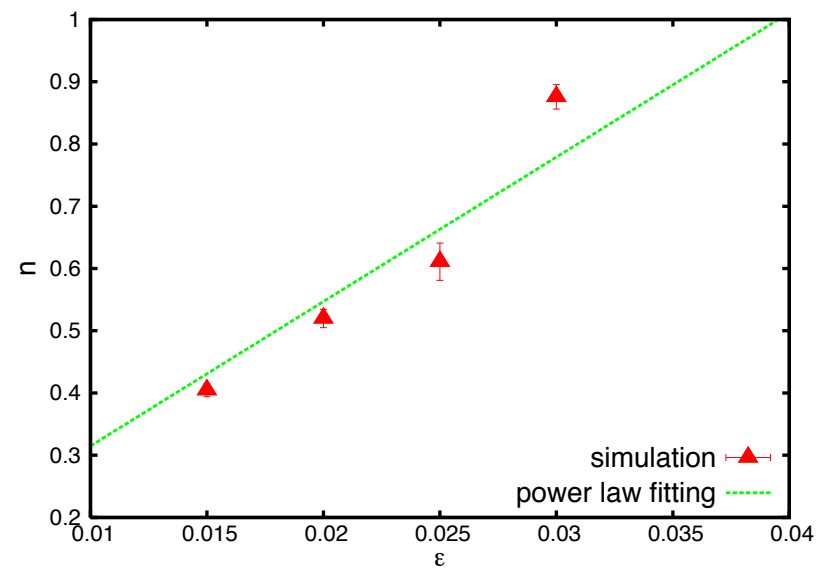

FIG. 6: The relation between the spectral index $n$ and the bias $\epsilon$. The dotted line represents the fitting formula given in Eq. (35).

Substituting Eqs. (33), (34), and (36) into Eq. (37), we obtain

$$
\left(\Omega_{\mathrm{gw}} h^{2}\right)_{\text {edge }} \simeq 3 \times 10^{-13} \times \epsilon^{-2} \lambda^{-2}\left(\frac{5.8 \epsilon}{\lambda}\right)^{20 \epsilon+0.08}\left(\frac{100}{g_{*}}\right)^{1 / 3}\left(\frac{\eta}{10^{17} \mathrm{GeV}}\right)^{4} .
$$

\section{Forecasts for Observations}

In the previous subsection, we found the fitting formulae given by Eqs. (33)- 36 ) and $(38)$ by combining the results of the numerical calculations and the analytic estimations. Now we use them to predict the gravitational wave spectrum for generic values of parameters. For simplicity we take $\lambda=1.0$ and $g_{*}=100$ in the following, then the spectrum depends on two parameters, the mass scale of the scalar field $\eta$ and the bias $\epsilon$. Note that $\epsilon$ must satisfy the condition given by Eq. (28) so that our results are applicable.

At a glance of the formulae (33)- $(38)$, we can say that $\eta$ determines the frequency of the spectrum and the strength of the amplitude, while $\epsilon$ determines the amplitude and the bandwidth of the gravitational wave spectrum. The large value of $\eta$ results in the appearance of the gravitational wave spectrum in high frequency range and large $\Omega_{\mathrm{gw}} h^{2}$. The small value of $\epsilon$ results in the broad band of spectrum and also large $\Omega_{\mathrm{gw}} h^{2}$. In Table [I, we show some examples of the value of various quantities estimated by using Eqs. (33)- $(38)$.

From Table I] we see that if the value of $\eta$ is around $10^{10}-10^{12} \mathrm{GeV}$, the edge would appear in the frequency band observable in future gravitational wave astronomy projects such as ET, BBO, and DECIGO. On the other hand, the peak of spectra appears in too high frequency to be observable. In Fig. 7) we show examples of gravitational wave spectra from domain walls estimated by using the formulae (33)-(38). We note that the maximum value of $\Omega_{\mathrm{gw}} h^{2}$ at the peak is smaller than the bound which comes from Big Bang Nucleosynthesis (BBN) [18, 48. We also show the contours of the frequency and the amplitude of the edge in the parameter space of $\eta$ and $\epsilon$ in Fig. 8. The parameter $\epsilon$ is constrained by the condition given by Eq. (28), and the white region in Fig. 8 is allowed by this constraint. The red region denoted by "Not scaling" is the parameter space in which walls disappear before they straighten up to horizon scale. The gray region denoted by "Wall domination" is the parameter space in which the energy density of domain walls dominates the total energy density of the universe. Note that the red region and the gray region do not imply that parameters in these regions are ruled out. In fact, domain walls can decay before reaching the scaling regime if $\epsilon$ is sufficiently large. Also, it might be possible that domain walls dominate the energy density of the universe if they disappear before the BBN epoch. However, we can use the formulae (33)-(38) only in the white region because in our analysis we assumed that domain walls straighten up to the horizon scale and disappear before they dominates the energy density of the universe. Especially, our numerical simulations are performed in the radiation dominated background, and we do not take into account the contribution of the energy density of domain walls. From Fig. 8 , we can say that if $\eta$ is around $10^{10}-10^{12} \mathrm{GeV}$, we would be able to measure the value of the scale of $\eta$ and the bias 


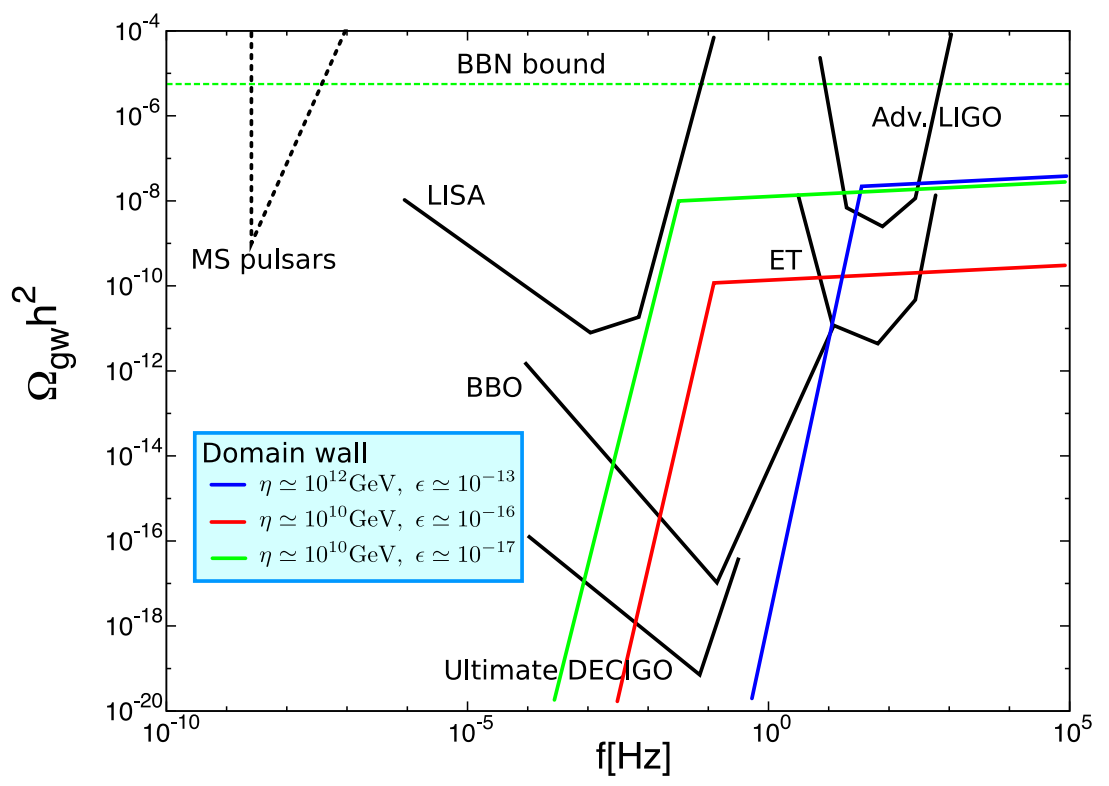

FIG. 7: The schematics of gravitational wave spectra from collapsing domain walls. We show three spectra for the case with $(\eta, \epsilon)=\left(10^{12} \mathrm{GeV}, 10^{-13}\right)$ (blue), $(\eta, \epsilon)=\left(10^{10} \mathrm{GeV}, 10^{-16}\right)$ (red), and $(\eta, \epsilon)=\left(10^{10} \mathrm{GeV}, 10^{-17}\right)$ (green). We also roughly plot the sensitivity of planned detectors, Advanced LIGO [44], LISA [23], ET [26], BBO [24, and Ultimate DECIGO [45]. The dotted lines represent the constraints obtained from the observation of millisec pulsars [46, 47] (black) and Big Bang Nucleosynthesis [18, 48] (green).

$\epsilon$ from the frequency and the amplitude of the edge of the gravitational wave spectra in future gravitational wave experiments.

Before going to the conclusion, we comment on the accuracy of the estimation we made. There are large uncertainties in the determination of the frequency dependence in Eq. (35) because of the limited dynamical range of the simulations. As we noted before, the uncertainty of the spectral index $n$ is about $50 \%$. This uncertainty might become larger when we extrapolate the result for other values of $\epsilon$. Even if we neglect this increase in the uncertainties and only take the uncertainty in the numerical result into account, we expect that the uncertainty in the magnitude of $\left(\Omega_{\mathrm{gw}} h^{2}\right)_{\text {edge }}$ for

TABLE I: The estimation of the amplitude and the frequency of the peak and the edge for various value of $\eta$. We also show the condition for $\epsilon$ given by Eq. (28). The upper line in each row corresponds to the value in the case where $\epsilon$ is taken as its minimum value, and the lower line corresponds to the value in the case where $\epsilon$ is taken as its maximum value. Note that domain walls with a low energy scale such as $\eta=100 \mathrm{GeV}$ and small $\epsilon$ might be ruled out by the pulsar timing experiments (see Fig. 7).

\begin{tabular}{cccccc}
\hline \hline$\eta$ & $f_{\text {peak }}$ & $\left(\Omega_{\mathrm{gw}} h^{2}\right)_{\text {peak }}$ & $f_{\text {edge }}$ & $\left(\Omega_{\mathrm{gw}} h^{2}\right)_{\text {edge }}$ & Condition for $\epsilon$ \\
\hline \hline \multirow{2}{*}{$10^{15} \mathrm{GeV}$} & $4.5 \times 10^{12} \mathrm{~Hz}$ & $2.1 \times 10^{-6}$ & $9.8 \times 10^{5} \mathrm{~Hz}$ & $6.0 \times 10^{-7}$ & \multirow{2}{*}{$3.7 \times 10^{-8}<\epsilon<3.8 \times 10^{-3}$} \\
& $1.4 \times 10^{10} \mathrm{~Hz}$ & $1.9 \times 10^{-16}$ & $3.1 \times 10^{8} \mathrm{~Hz}$ & $1.1 \times 10^{-16}$ & \\
\hline \multirow{2}{*}{$10^{12} \mathrm{GeV}$} & $1.4 \times 10^{14} \mathrm{~Hz}$ & $2.1 \times 10^{-6}$ & $31 \mathrm{~Hz}$ & $2.0 \times 10^{-7}$ & \multirow{2}{*}{$3.7 \times 10^{-14}<\epsilon<3.8 \times 10^{-6}$} \\
& $1.4 \times 10^{10} \mathrm{~Hz}$ & $1.9 \times 10^{-22}$ & $3.1 \times 10^{5} \mathrm{~Hz}$ & $8.2 \times 10^{-23}$ & \\
\hline \multirow{2}{*}{$10^{10} \mathrm{GeV}$} & $1.4 \times 10^{15} \mathrm{~Hz}$ & $2.1 \times 10^{-6}$ & $3.1 \times 10^{-2} \mathrm{~Hz}$ & $9.5 \times 10^{-8}$ & \multirow{2}{*}{$3.7 \times 10^{-18}<\epsilon<3.8 \times 10^{-8}$} \\
& $1.4 \times 10^{10} \mathrm{~Hz}$ & $1.9 \times 10^{-26}$ & $3.1 \times 10^{3} \mathrm{~Hz}$ & $5.7 \times 10^{-27}$ & \\
\hline \multirow{2}{*}{$100 \mathrm{GeV}$} & $1.4 \times 10^{19} \mathrm{~Hz}$ & $2.1 \times 10^{-6}$ & $3.1 \times 10^{-14} \mathrm{~Hz}$ & $5.0 \times 10^{-9}$ & \multirow{2}{*}{$3.7 \times 10^{-34}<\epsilon<3.8 \times 10^{-16}$} \\
& $1.4 \times 10^{10} \mathrm{~Hz}$ & $1.9 \times 10^{-42}$ & $3.1 \times 10^{-5} \mathrm{~Hz}$ & $1.3 \times 10^{-43}$ & \\
\hline \hline
\end{tabular}


the forecast in Fig. 7 can be a factor of $\mathcal{O}\left(10^{ \pm 1}\right)$. In order to improve the accuracy of the estimation, we have to run simulations with a much larger dynamical range and with many realizations.

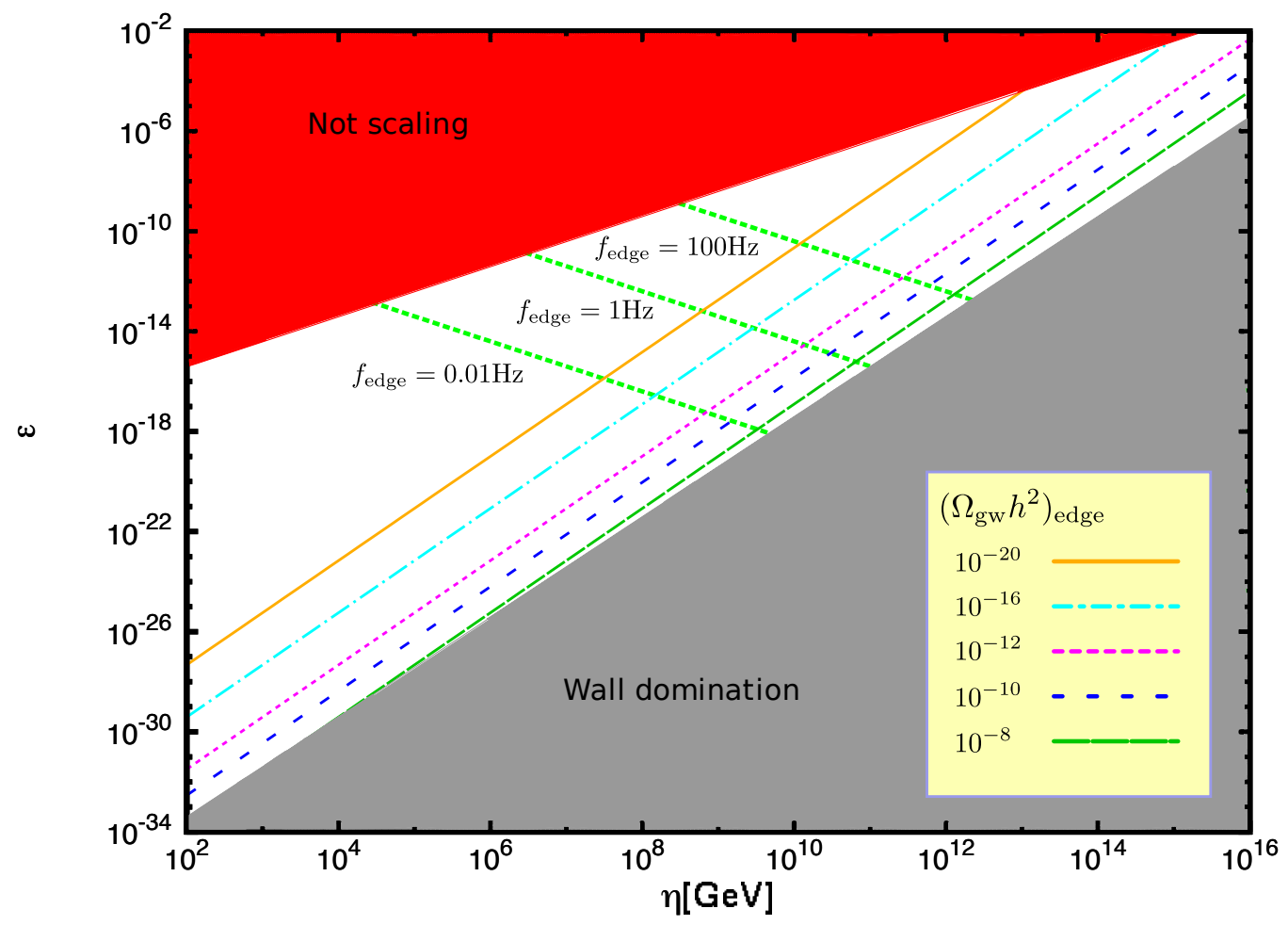

FIG. 8: The contours of the frequency and the amplitude of the edge in the parameter space of $\eta$ and $\epsilon$ (we fixed $\lambda=1.0$ and $\left.g_{*}=100\right)$. The gray region corresponds to the case that the energy density of domain walls dominates the total energy density of the universe [the left hand side of Eq. [28)]. The red region corresponds to the case that $\epsilon$ is too large to form domain walls with scaling phase [the right hand side of Eq. [28)]. The dotted green line shows the frequency $f_{\text {edge }}=0.01 \mathrm{~Hz}, 1 \mathrm{~Hz}$, and $100 \mathrm{~Hz}$. We also show the line with the amplitudes of the edge, $\Omega_{\mathrm{gw}} h^{2}=10^{-20}$ (orange), $10^{-16}$ (light blue), $10^{-12}$ (pink), $10^{-10}$ (blue), and $10^{-8}$ (light green).

\section{CONCLUSION}

In this paper, we have studied gravitational waves from domain walls with approximate $Z_{2}$ symmetry. We have performed three dimensional lattice simulations and followed the process of creation, evolution and collapse of domain walls. We have confirmed that stable domain walls evolve to maintain the scaling solution, and biased domain walls annihilate in the time scale estimated by Eq. (11). In numerical simulations we have solved the field equation directly rather than using the standard PRS method. It is found that the spectrum of gravitational waves extends over broad frequency band, contrary to the naive expectation that gravitational waves have a peak at the frequency corresponding to the horizon scale as conjectured in [17, 27]. The results of numerical simulations have revealed that the spectrum has the edge corresponding to the horizon scale at the time when walls collapse and the peak corresponding to the small scale on the wall typically given by the wall width. In the intermediate range between edge and peak, the gravitational wave amplitude increases moderately with frequency, $\Omega_{\mathrm{gw}} h^{2} \sim f^{0.08}$.

By combining analytic estimations and numerical results, we have obtained the formulae (33)-(36) and (38) for the frequency and the amplitude of gravitational wave spectra. We have extrapolated the results to more generic parameter space and showed that the edge of the gravitational wave spectrum from domain walls with energy scale 
$\eta=10^{10}-10^{12} \mathrm{GeV}$ is observable in future gravitational wave experiments such as ET, BBO, and DECIGO. Therefore, we expect that the gravitational waves from the collapse of domain walls can be a new observational window to probe the models of high energy physics which have (approximate) discrete symmetry. This observation may enable us to search the unknown physics through the estimation of the parameters such as bias and provide rich information about the theory beyond the standard model of particle physics.

\section{Acknowledgments}

We would like to thank Kazunori Nakayama and Fuminobu Takahashi for useful discussions. This work is supported by Grant-in-Aid for Scientific research from the Ministry of Education, Science, Sports, and Culture (MEXT), Japan, No.14102004 and No.21111006 (M.K.) and also by World Premier International Research Center Initiative (WPI Initiative), MEXT, Japan.

\section{Appendix A: Numerical Calculations}

In this appendix we describe the setup for our numerical computations.

\section{Formulation}

We adopt the formulation similar to that of Yamaguchi et al. [49, 50, for the simulation of global strings. The time, the Hubble radius, and the temperature are related by the Friedmann equation in the radiation dominated universe

$$
t=\frac{1}{2 H}=\frac{\xi}{T^{2}}
$$

where $\xi$ is the constant defined by $\xi \equiv\left(45 M_{P}^{2} / 16 \pi^{2} g_{*}\right)^{1 / 2} \simeq 3.68 \times 10^{17}\left(g_{*} / 100\right)^{-1 / 2} \mathrm{GeV}$. We choose the initial time $t_{i}$ so that the temperature at $t=t_{i}$ is twice of the critical temperature of phase transition, $T_{i}=2 T_{c}=4 \eta$. We normalize the dimensionful quantities in the unit of $t_{i}$. For example, $t \rightarrow t / t_{i}, x \rightarrow x / t_{i}, \phi \rightarrow \phi t_{i}$, etc. It is useful to define the dimensionless quantity $\zeta \equiv \xi / \eta$. By using $\zeta$, equation of motion for the scalar field renormalized by $t_{i}$ can be written as

$$
\begin{gathered}
\ddot{\phi}+\frac{3}{2 t} \dot{\phi}-\frac{1}{t} \nabla^{2} \phi+\frac{d V}{d \phi}=0 \\
\frac{d V}{d \phi}=\left(\lambda \phi+\epsilon \frac{\zeta}{16}\right)\left(\phi^{2}-\frac{\zeta^{2}}{256}\right)+\frac{\lambda \zeta^{2}}{64 t} \phi .
\end{gathered}
$$

We choose the value of $\zeta$ as 3.5 which corresponds to $\eta=1.05 \times 10^{17} \mathrm{GeV}$.

The (comoving) size of the simulation box is chosen to be $b$ in the unit of $t_{i}$, and the lattice spacing is $\delta x=b / N$ where $N$ is the number of grid points. Here we take $N=256$. The ratio between the Hubble horizon scale and the physical lattice spacing is

$$
\frac{H^{-1}}{\delta x_{\text {phys }}}=\frac{2 N}{b}\left(t / t_{i}\right)^{1 / 2},
$$

and the ratio between the wall width and the physical lattice spacing is

$$
\frac{\delta_{w}}{\delta x_{\text {phys }}}=\frac{16 N}{\lambda^{1 / 2} \zeta b}\left(t / t_{i}\right)^{-1 / 2} .
$$

At the final time $t=151 t_{i}$, these ratios become $H^{-1} / \delta x_{\text {phys }} \simeq 252<N, \delta_{w} / \delta x_{\text {phys }} \simeq 3.81$ for $b=25$, and $H^{-1} / \delta x_{\text {phys }} \simeq 126<N, \delta_{w} / \delta x_{\text {phys }} \simeq 1.90$ for $b=50$. Therefore, the resolution of the simulations is sufficiently good even at the final time.

We put the periodic boundary condition in the configuration of the scalar field. We solve the time evolution by using the fourth order Runge-Kutta method. 


\section{Initial Conditions}

As the initial conditions, we assume that the quantum field $\phi$ is in thermal equilibrium with temperature $T_{i} \equiv 1 / \beta$. The field and its derivative satisfy the equal-time correlation relations

$$
\begin{aligned}
\langle\beta|\phi(x) \phi(y)| \beta\rangle_{\text {equal-time }} & =\int \frac{d^{3} k}{(2 \pi)^{3}} \frac{1}{2 E_{k}}\left[1+2 n_{k}\right] e^{i \mathbf{k} \cdot(\mathbf{x}-\mathbf{y})}, \\
\langle\beta|\dot{\phi}(x) \dot{\phi}(y)| \beta\rangle_{\text {equal-time }} & =\int \frac{d^{3} k}{(2 \pi)^{3}} \frac{E_{k}}{2}\left[1+2 n_{k}\right] e^{i \mathbf{k} \cdot(\mathbf{x}-\mathbf{y})},
\end{aligned}
$$

where $E_{k}=\sqrt{k^{2}+m^{2}}$ and $n_{k}$ is the occupation number of the Bose-Einstein distributions, $n_{k}=\left(e^{E_{k} / T_{i}}-1\right)^{-1}$. The mass of the scalar field is given by

$$
m^{2}=\left.\frac{d^{2} V}{d \phi^{2}}\right|_{\phi=0}=\frac{\lambda}{4}\left(T_{i}^{2}-4 \eta^{2}\right)=3 \lambda \eta^{2} .
$$

The first term in the square bracket of Eqs. (A6) and A7) corresponds to the vacuum fluctuations which contribute as a divergent term when we perform the integral of $k$. Hence we subtract this term and use the renormalized correlation functions

$$
\begin{aligned}
\langle\beta|\phi(x) \phi(y)| \beta\rangle_{\text {ren. }} & =\int \frac{d^{3} k}{(2 \pi)^{3}} \frac{n_{k}}{E_{k}}, \\
\langle\beta|\dot{\phi}(x) \dot{\phi}(y)| \beta\rangle_{\text {ren. }} & =\int \frac{d^{3} k}{(2 \pi)^{3}} E_{k} n_{k} .
\end{aligned}
$$

In the momentum space, these correlation functions can be written as

$$
\begin{aligned}
& \left\langle\beta\left|\tilde{\phi}(\mathbf{k}) \tilde{\phi}\left(\mathbf{k}^{\prime}\right)\right| \beta\right\rangle_{\text {ren. }}=\frac{n_{k}}{E_{k}}(2 \pi)^{3} \delta^{(3)}\left(\mathbf{k}+\mathbf{k}^{\prime}\right), \\
& \left\langle\beta\left|\dot{\tilde{\phi}}(\mathbf{k}) \dot{\tilde{\phi}}\left(\mathbf{k}^{\prime}\right)\right| \beta\right\rangle_{\text {ren. }}=E_{k} n_{k}(2 \pi)^{3} \delta^{(3)}\left(\mathbf{k}+\mathbf{k}^{\prime}\right),
\end{aligned}
$$

where $\tilde{\phi}(\mathbf{k})$ is the Fourier transform of $\phi(\mathbf{x})$. Since $\tilde{\phi}(\mathbf{k})$ and $\dot{\tilde{\phi}}(\mathbf{k})$ are uncorrelated in the momentum space, we generate $\tilde{\phi}(\mathbf{k})$ and $\dot{\tilde{\phi}}(\mathbf{k})$ in the momentum space randomly following the Gaussian distribution with

$$
\begin{gathered}
\left\langle|\tilde{\phi}(\mathbf{k})|^{2}\right\rangle=\frac{n_{k}}{E_{k}} V, \quad\left\langle|\dot{\tilde{\phi}}(\mathbf{k})|^{2}\right\rangle=n_{k} E_{k} V, \\
\langle\tilde{\phi}(\mathbf{k})\rangle=\langle\dot{\tilde{\phi}}(\mathbf{k})\rangle=0 .
\end{gathered}
$$

Then we transform them into the configuration space and obtain the initial field configurations $\phi(\mathbf{x})$ and $\dot{\phi}(\mathbf{x})$. Here we used $(2 \pi)^{3} \delta^{(3)}(0) \simeq V$, where $V=b^{3} t_{i}^{3}$ is the comoving volume of the simulation box.

\section{Calculation of the Area Density}

For calculation of the area density of domain walls, we use the algorithm introduced by Press, Ryden, and Spergel [28. Let us call the neighboring grid points that differ by one in $x, y, z$ location as the "link". Define the quantity $\delta_{ \pm}$which takes the value 1 if $\phi$ has different signs at the two ends of a link and the value 0 if $\phi$ has the same sign at the two ends of a link. We evaluate the area density $A / V$ by summing up $\delta_{ \pm}$for each of the links with the weighting factor

$$
A / V=C \sum_{\text {links }} \delta_{ \pm} \frac{|\nabla \phi|}{\left|\phi_{, x}\right|+\left|\phi_{, y}\right|+\left|\phi_{, z}\right|},
$$

where $\phi_{, x}$, etc. is a derivative of $\phi(\mathbf{x})$ with respect to $x$, and $C$ is a normalization coefficient. We choose $C$ such that $A / V=1$ is satisfied when all the links have the value $\delta_{ \pm}=1$.

[1] T. W. B. Kibble, Journal of Physics A Mathematical General 9, 1387 (1976). 
[2] A. Vilenkin and E. P. S. Shellard, Cosmic strings and other topological defects (Cambridge University Press, 1994).

[3] G. B. Gelmini, M. Gleiser, and E. W. Kolb, Phys. Rev. D 39, 1558 (1989).

[4] Y. B. Zel'Dovich, I. Y. Kobzarev, and L. B. Okun', Soviet Journal of Experimental and Theoretical Physics 40, 1 (1974).

[5] B. Rai and G. Senjanović, Phys. Rev. D 49, 2729 (1994), arXiv:hep-ph/9301240.

[6] A. Vilenkin, Phys. Rev. D 23, 852 (1981).

[7] P. Sikivie, Physical Review Letters 48, 1156 (1982).

[8] A. K. Mohanty and F. W. Stecker, Physics Letters B 143, 351 (1984).

[9] Z. Lalak, B. A. Ovrut, and S. Thomas, Phys. Rev. D 51, 5456 (1995).

[10] D. Coulson, Z. Lalak, and B. Ovrut, Phys. Rev. D 53, 4237 (1996).

[11] S. W. Hawking, I. G. Moss, and J. M. Stewart, Phys. Rev. D 26, 2681 (1982).

[12] M. S. Turner and F. Wilczek, Physical Review Letters 65, 3080 (1990).

[13] A. Kosowsky, M. S. Turner, and R. Watkins, Phys. Rev. D 45, 4514 (1992).

[14] A. Kosowsky, M. S. Turner, and R. Watkins, Physical Review Letters 69, 2026 (1992).

[15] A. Kosowsky and M. S. Turner, Phys. Rev. D 47, 4372 (1993), arXiv:astro-ph/9211004.

[16] M. Kamionkowski, A. Kosowsky, and M. S. Turner, Phys. Rev. D 49, 2837 (1994), arXiv:astro-ph/9310044.

[17] M. Gleiser and R. Roberts, Physical Review Letters 81, 5497 (1998), arXiv:astro-ph/9807260.

[18] M. Maggiore, Phys. Rep. 331, 283 (2000), arXiv:gr-qc/9909001.

[19] M. Maggiore, Gravitational Waves Volume 1: Theory and Experiments (Oxford University Press, 2008).

[20] B. S. Sathyaprakash and B. F. Schutz, Living Reviews in Relativity 12, 2 (2009), 0903.0338.

[21] A. Abramovici, W. E. Althouse, R. W. P. Drever, Y. Gursel, S. Kawamura, F. J. Raab, D. Shoemaker, L. Sievers, R. E. Spero, and K. S. Thorne, Science 256, 325 (1992).

[22] K. Kuroda, M. Ohashi, S. Miyoki, H. Ishizuka, C. T. Taylor, K. Yamamoto, O. Miyakawa, M. Fujimoto, S. Kawamura, R. Takahashi, et al., Classical and Quantum Gravity 19, 1237 (2002).

[23] URL http://lisa.nasa.gov/

[24] J. Crowder and N. J. Cornish, Phys. Rev. D 72, 083005 (2005), arXiv:gr-qc/0506015.

[25] S. Kawamura, T. Nakamura, M. Ando, N. Seto, K. Tsubono, K. Numata, R. Takahashi, S. Nagano, T. Ishikawa, M. Musha, et al., Classical and Quantum Gravity 23, 125 (2006).

[26] URL http://www.et-gw.eu/.

[27] F. Takahashi, T. T. Yanagida, and K. Yonekura, Physics Letters B 664, 194 (2008), 0802.4335.

[28] W. H. Press, B. S. Ryden, and D. N. Spergel, ApJ 347, 590 (1989).

[29] L. Kawano, Phys. Rev. D 41, 1013 (1990).

[30] Y. Nambu, in Symmetries and Quark Models, edited by R. Chand (1970), pp. 269-+.

[31] T. Gotō, Progress of Theoretical Physics 46, 1560 (1971).

[32] T. Garagounis and M. Hindmarsh, Phys. Rev. D 68, 103506 (2003), arXiv:hep-ph/0212359.

[33] J. C. Oliveira, C. J. Martins, and P. P. Avelino, Phys. Rev. D 71, 083509 (2005), arXiv:hep-ph/0410356.

[34] P. P. Avelino, J. C. R. E. Oliveira, and C. J. A. P. Martins, Physics Letters B 610, 1 (2005), arXiv:hep-th/0503226.

[35] S. E. Larsson, S. Sarkar, and P. L. White, Phys. Rev. D 55, 5129 (1997), arXiv:hep-ph/9608319.

[36] M. Hindmarsh, Physical Review Letters 77, 4495 (1996), arXiv:hep-ph/9605332.

[37] M. Hindmarsh, Phys. Rev. D 68, 043510 (2003), arXiv:hep-ph/0207267.

[38] P. P. Avelino, C. J. A. P. Martins, and J. C. R. E. Oliveira, Phys. Rev. D 72, 083506 (2005), arXiv:hep-ph/0507272.

[39] D. Stauffer, Phys. Rep. 54, 1 (1979).

[40] J. Dufaux, A. Bergman, G. Felder, L. Kofman, and J. Uzan, Phys. Rev. D 76, 123517 (2007), 0707.0875.

[41] J. Dufaux, G. Felder, L. Kofman, and O. Navros, Journal of Cosmology and Astro-Particle Physics 3, 1 (2009), 0812.2917.

[42] L. Kofman, A. Linde, and A. A. Starobinsky, Physical Review Letters 73, 3195 (1994), arXiv:hep-th/9405187.

[43] L. Kofman, A. Linde, and A. A. Starobinsky, Phys. Rev. D 56, 3258 (1997), arXiv:hep-ph/9704452.

[44] URL http://www.ligo.caltech.edu/advLIGO/scripts/summary.shtml.

[45] N. Seto, S. Kawamura, and T. Nakamura, Physical Review Letters 87, 221103 (2001), arXiv:astro-ph/0108011.

[46] V. M. Kaspi, J. H. Taylor, and M. F. Ryba, ApJ 428, 713 (1994).

[47] A. N. Lommen, in Neutron Stars, Pulsars, and Supernova Remnants, edited by W. Becker, H. Lesch, \& J. Trümper (2002), pp. $114-+$.

[48] B. Allen, in Some Topics on General Relativity and Gravitational Radiation, edited by J. A. Miralles, J. A. Morales, \& D. Saez (1997), pp. 3-+, arXiv:gr-qc/9604033.

[49] M. Yamaguchi, J. Yokoyama, and M. Kawasaki, Progress of Theoretical Physics 100, 535 (1998), arXiv:hep-ph/9808326.

[50] M. Yamaguchi and J. Yokoyama, Phys. Rev. D 67, 103514 (2003), arXiv:hep-ph/0210343. 